Canadian

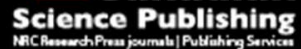

Canadian Journal of Physiology and Pharmacology Revue canadienne de physiologie et pharmacologie

\title{
Sex-specific vascular responses of the rat aorta: Effects of moderate term (intermediate stage) streptozotocin-induced diabetes
}

\begin{tabular}{|r|l|}
\hline Journal: & Canadian Journal of Physiology and Pharmacology \\
\hline Manuscript ID & cjpp-2015-0272.R3 \\
\hline Danuscript Type: & Article \\
\hline Complete List of Authors: & \begin{tabular}{rl|} 
Han, Xiaoyuan; University of the Pacific, Physiology and Pharmacology, \\
Thomas J. Long School of Pharmacy and Health Sciences \\
Shaligram, Sonali; University of the Pacific, Physiology and Pharmacology, \\
Thomas J. Long School of Pharmacy and Health Sciences \\
Zhang, Rui; University of the Pacific, Physiology and Pharmacology, \\
Thomas J. Long School of Pharmacy and Health Sciences \\
Anderson, Leigh; University of the Pacific, Department of Biomedical \\
Sciences, Arthur A. Dugoni School of Dentistry \\
Rahimian, Roshanak; University of the Pacific, Physiology and \\
Pharmacology, Thomas J. Long School of Pharmacy and Health Sciences
\end{tabular} \\
\hline Keyword: & $\begin{array}{l}\text { Sex Differences, Endothelial function, Nitric Oxide, Streptozotocin-induced } \\
\text { diabetes, Rat Aorta }\end{array}$ \\
\hline &
\end{tabular}

\section{SCHOLARONE ${ }^{m}$ \\ Manuscripts}


Sex-specific vascular responses of the rat aorta: Effects of moderate term (intermediate stage) streptozotocin-induced diabetes

Xiaoyuan Han ${ }^{1}$, Sonali Shaligram ${ }^{1}$, Rui Zhang ${ }^{1}$, Leigh Anderson ${ }^{2}$ and Roshanak Rahimian $^{1 *}$

${ }^{1}$ Department of Physiology \& Pharmacology, Thomas J. Long School of Pharmacy \& Health Sciences, University of the Pacific, Stockton, CA 95211

${ }^{2}$ Department of Biomedical Sciences, Arthur A. Dugoni School of Dentistry, University of the Pacific, San Francisco, CA 94115

* To whom correspondence should be addressed: Roshanak Rahimian Ph. D., Department of Physiology \& Pharmacology, Thomas J. Long School of Pharmacy \& Health Sciences, University of the Pacific, 3601 Pacific Ave, Stockton, CA 95211. Telephone: (209) 946-2373, Fax: (209) 946-2857, Email: rrahimian@pacific.edu 


\begin{abstract}
Hyperglycemia affects male and female vascular beds differently. We have previously shown that 1 week after the induction of diabetes with streptozotocin (STZ) male and female rats exhibit differences in aortic endothelial function. To examine this phenomenon further, aortic responses were studied in female and male rats 8 weeks after the induction of diabetes (intermediate stage). Endothelium-dependent vasodilatation (EDV) to acetylcholine (ACh) was measured in phenylephrine (PE) pre-contracted rat aortic rings. Concentration response curves to PE were generated before and after LNAME, a nitric oxide synthase (NOS) inhibitor. Furthermore, mRNA expression of endothelial nitric oxide synthase (eNOS) and NADPH oxidase subunit (Nox1) were determined. At 8 week, diabetes impaired EDV to a greater extent in female than male aortae. Furthermore, the responsiveness to PE was significantly enhanced only in female diabetic rats, and basal NO, as indicated by the potentiation of the response to PE after LNAME, was reduced in female diabetic rat aortas to the same levels as in males. In addition, eNOS mRNA expression was decreased, while the Nox1 expression was significantly enhanced in diabetic female rats. These results suggest that aortic function in female diabetic rats after 8 weeks exhibits a more prominent impairment and that NO may be involved.
\end{abstract}

\title{
Key words:
}

Sex Differences; Endothelial function; Nitric Oxide, Streptozotocin (STZ); Diabetes; Rat Aorta 


\section{INTRODUCTION}

Cardiovascular diseases (CVD) are a main cause of morbidity and mortality in diabetes, and both micro- and macrovascular complications play a major role in the development of CVD in diabetic patients. Endothelial dysfunction is a hallmark of the diabetic vascular disease; it is defined as a reduced endothelium-dependent vasodilation (EDV) to vasodilators, such as acetylcholine (ACh) and bradykinin, or flow-mediated vasodilation. Thus, EDV is generally used as a reproducible parameter to investigate endothelial function under various pathological conditions. A number of reports including our previous studies have shown that acute exposure of blood vessels to high glucose impairs EDV to ACh (Goel et al. 2008; Goel et al. 2007). Impaired EDV has been also reported in experimental models of diabetes (De Vriese et al. 2000; Johnstone et al. 1993; Zhang et al. 2012). However, there are also reports demonstrating an enhanced EDV in diabetes (Bhardwaj and Moore 1988; Shen et al. 2003), and alterations in EDV are dependent on the duration of the diabetic state (Clarkson et al. 1996; Pieper 1999).

Previously, we reported that EDV in mesenteric arteries was impaired at early stage of diabetes ( 1 week duration) regardless of sexes. However, 8 weeks after the induction of diabetes (an intermediate stage) the extent of impairment was greater in females than in males (Zhang et al. 2012). We also observed sex differences in the development of impaired EDV in rat aorta one week after streptozotocin (STZ)-diabetes was induced (Han et al. 2014). Thus, we examined the role of sex on the development of abnormal aortic responses in female and male STZ diabetic rats after 8 weeks. 
EDV is dependent on a variety endothelial derived relaxing factors (EDRFs), such as nitric oxide (NO), prostacyclin and endothelial derived hyperpolarizing factors (EDHF). NO is the dominant EDRF in large conduit arteries, such as the aorta (Shimokawa et al. 1996). NO bioavailability is determined by the balance between synthesis and degradation of the molecule. Thus endothelial NO bioavailability is regulated at mainly two levels: 1) eNOS gene expression/basal NO production and 2) degradation of NO by reactive oxygen species (ROS) including superoxide. NADPH oxidase (Nox) is one of the major sources of superoxide in the cardiovascular system (Cai et al. 2003; Gorlach et al. 2000; Griendling et al. 2000). Among Nox subunits, Nox1 is mainly expressed in large conduit arteries (Lassegue et al. 2001). The impact of diabetes on eNOS expression is contentious, with studies reporting reductions (Fu et al. 2007; Olukman et al. 2010), or an increase (Ikubo et al. 2011; Kazuyama et al. 2009) in rat aortae. Thus, we also investigated whether sex changes the basal NO along with eNOS and Nox1 mRNA expression in rat aorta at intermediate stage of STZ diabetes.

\section{MATERIALS AND METHODS}

\section{Materials}

All chemicals were purchased from Sigma Chemical Co. (St. Louis, MO, USA). All agents were dissolved in water, unless otherwise stated.

\section{Animals}

All animal protocols were approved by the Animal Care Committee of the University of the Pacific and complied with the Guide for the Care and Use of Laboratory 
Animals: Eighth Edition (2011). Euthanasia was carried out according to the recommendations from the 2013 AVMA Guidelines on Euthanasia and the NIH Guidelines for the Care and Use of Laboratory Animals.

Twenty four age-matched male and female Sprague-Dawley rats, 10-11 weeks of age (Simonsen Laboratories, Gilroy, CA) were randomly divided into four experimental groups of six animals in each group: control female, diabetic female, control male, and diabetic male. At the time, there were three additional age-matched female rats at the facility which were also included as control females for the ACh study.

After an overnight fast, diabetes was induced by single injection of STZ (60 $\mathrm{mg} / \mathrm{kg}$, i.v.) (Malhotra et al. 1981). Age-matched control animals were injected with a similar volume of citrate buffer. Only animals demonstrating non-fasting glucose levels higher than $300 \mathrm{mg} / \mathrm{dl}$ were considered diabetic. One of the diabetic female rats was excluded from the study as it exhibited the lethargic behavior following the tail vein injection of STZ. Rats were euthanized 8 weeks after STZ treatment using carbon dioxide $\left(\mathrm{CO}_{2}\right)$ euthanasia, and blood glucose levels and body weights were measured.

\section{Measurement of arterial tension}

The thoracic aorta was removed and placed in cold Krebs buffer (in mM: 119 $\mathrm{NaCl}, 4.7 \mathrm{KCl}, 1.18 \mathrm{KH}_{2} \mathrm{PO}_{4}, 1.17 \mathrm{MgSO}_{4}, 24.9 \mathrm{NaHCO}_{3}, 0.023 \mathrm{EDTA}, 1.6 \mathrm{CaCl}_{2}$, and 6 glucose) (Rahimian et al. 1997). Aortae were cleaned of fatty and adhering connective tissues and then cut into $2 \mathrm{~mm}$ rings. To measure isometric tension, the rings were suspended horizontally between two stainless steel hooks in individual organ baths containing $20 \mathrm{ml}$ of Krebs buffer at $37^{\circ} \mathrm{C}$ bubbled with $95 \% \mathrm{O}_{2}-5 \% \mathrm{CO}_{2}$. Isometric 
tension was continuously monitored with a computer based data acquisition system (PowerLab, ADInstruments, Colorado Springs, USA). The rings were equilibrated for 40 min under a resting tension of $1 \mathrm{~g}$ to allow development of a stable basal tone.

Stimulation of rings with $80 \mathrm{mM} \mathrm{KCl}$ was repeated two times every 20 min to induce maximum contraction. To test integrity of endothelium, aortic rings were pre-contracted with phenylephrine $(\mathrm{PE}, 2 \mu \mathrm{M})$ that produced $80 \%$ of maximum response and a single dose of ACh $(10 \mu \mathrm{M})$ was used to relax the aortic rings. The ability of acetylcholine (ACh, $10 \mu \mathrm{M})$ to induce relaxation in PE $(2 \mu \mathrm{M})$ pre-contracted vessels was taken as evidence of a viable endothelium.

\section{Relaxation responses to $\mathrm{ACh}$}

Aortic rings from control and age-matched male and female diabetic rats were contracted with PE $(2 \mu \mathrm{M})$. Dilator response curves were obtained by the addition of increasing concentrations of $\mathrm{ACh}\left(10^{-8}\right.$ to $\left.10^{-5} \mathrm{M}\right)$. Tissues were washed with Krebs' solution for $30 \mathrm{~min}$ to allow relaxation to basal tone.

\section{Relaxation responses to sodium nitroprusside (SNP)}

Responses to sodium nitroprusside $\left(10^{-9}\right.$ to $\left.10^{-5} \mathrm{M}\right)$, an endothelium-independent vasodilating agent, were generated in a separate set or aortic rings pre-contracted with PE $(2 \mu \mathrm{M})$. ACh- and SNP-induced relaxations were expressed as the percent relaxation from maximum PE contraction at each concentration. Similarly, the recorded increase in the force of contraction was calculated as the percent maximum contraction obtained with $\mathrm{PE}$ at the highest dose. EC50, the concentration of the agonist that produced half 
maximal effect (Emax), which was calculated by a sigmoidal dose-response, model (for variable slope) using GraphPad Prism 5.01 (GraphPad Software Inc.). The sensitivity of the agonists was expressed as $\mathrm{pD}_{2}$ values $\left(-\log \left[\mathrm{EC}_{50}\right]\right)$, which were normally distributed.

\section{Contractile responses to $\mathrm{PE}$}

Contractile responses $(\mathrm{CRC})$ to $\mathrm{PE}\left(10^{-8}\right.$ to $\left.10^{-5} \mathrm{M}\right)$ were obtained before and after incubation with $\mathrm{N}^{\omega}$-Nitro-L-arginine methyl ester (L-NAME, $200 \mu \mathrm{M}$ ), a NOS inhibitor, in the presence of indomethacin (indo, $10 \mu \mathrm{M}$, dissolved in DMSO), a cyclooxygenase (COX) inhibitor. The use of this concentration of L-NAME was based on previous studies (Han et al. 2014; Zhang et al. 2012). A vehicle only (no drugs present) study was performed simultaneously in aortic rings from the same animal. No difference between the first and second CRC to PE was observed (data not shown). The area under the curve (AUC) was determined using GraphPad Prism 5.01 with trapezoidal method. To compare the effect of pharmacological agents, such as L-NAME on PE response, results were expressed as differences in area under the concentration-response curve ( $\triangle A U C)$ in control (absence of drug) and experimental (presence of drug) conditions.

\section{Real-time PCR}

Thoracic aortae was isolated as described above and cut into $12 \mathrm{~mm}$ segments. RNA was extracted from segments using RNeasy mini kit (QIAGEN). First-strand cDNA was synthesized by reverse transcription of $2 \mu \mathrm{g}$ of total RNA using the Omniscript RT kit (QIAGEN) in a total volume of $20 \mu 1$, according to the manufacturer's instructions. The gene fragments were then specifically amplified with iQ SYBR Green 
Supermix (Bio-Rad) using real-time RT-PCR (MyiQ Single-Color Real-Time PCR Detection System, Bio-Rad). Internal variations were normalized to rat glyceraldehyde 3-phosphate dehydrogenase (GAPDH) or $\beta$-actin, and expression was analyzed by $2^{-\Delta \Delta C t}$ method (Livak and Schmittgen 2001). The following primers were used for detection of gene expression: 5'-TGG GTG TGA ACC ACA AGA AA-3' (forward) and 5'-GTG GCA GTG ATG ACA TGG AC-3' (reverse) for rat GAPDH; 5'-CTG GGT ATG GAA TCC TGT GG-3' (forward) and 5'-TCA TCG TAC TCC TGC TTG CTG-3' for rat $\beta$ actin; 5'- ACT GCG TCG CTT CAT TAG GT-3' (forward) and 5'- TAG GCA AGC GCT TTA CCA CT-3' (reverse) for rat eNOS; 5'- GGC AAC ATG AGA GCT GCA TA-3' (forward) and 5'- GCA AGT GTC AAC CAG CAA GA-3'(reverse) for rat Nox1. Specificity was verified by electrophoresis of the PCR products on a $2 \%$ agarose gel stained with ethidium bromide.

\section{Statistical analysis}

Data were reported as the mean \pm standard error of the mean (SEM), and were analyzed using SPSS software (SPSS Inc.). To compare groups, we used a two-way analysis of variance (ANOVA), using sex and diabetes as factors, and included their interaction. Comparison of response curves in a pre/post-test format (before and after a drug) within a group was done using ANOVA with repeated measures. Three-way ANOVA with factors being sex, diabetes and drugs were used to compare among group means in Table 3. The significance level was set to $\alpha=0.05$, followed by a post hoc test, as appropriate. 


\section{RESULTS}

\section{1) Effects of sex and diabetes on rat body weight and blood glucose}

A significant difference in the final weights of control males and females (396.8 \pm 11.9 vs. $252.8 \pm 3.4 \mathrm{~g})$ was expected, as they were age-matched (Table 1). Although non-fasting blood glucose levels were approximately $30 \%$ lower in control males than in control females $(124 \pm 12$ vs. $161 \pm 29 \mathrm{mg} / \mathrm{dl})$, the difference was not statistically significant. It is not known why control female rats have higher non-fasting glucose levels, but this sex difference is in accordance with the observation of Faerch et al. showing that women have higher $2 \mathrm{~h}$ post-OGTT plasma glucose (Faerch et al. 2010). Faerch et al. speculated that the higher $2 \mathrm{~h}$ post-OGTT plasma glucose in women may be related to differences in body size between men and women.

The body weights of both female and male STZ-induced diabetic rats were lower than those of age-matched non-diabetic controls (Table 1). Non-fasting blood glucose levels of diabetic female and male rats were significantly higher than those of their respective non-diabetic controls (Table 1).

\section{2) Effects of sex and diabetes on $\mathrm{ACh}$-induced relaxation in rat aorta}

A sex difference was observed in aortic relaxation responses to ACh in agematched non-diabetic control rats (Figure 1). Both sensitivity, as assessed by $-\log \left[\mathrm{EC}_{50}\right]$ $\left(\mathrm{pD}_{2}\right)$ value, and Emax to ACh were higher in females than those in males (Table 2). Eight weeks after the induction of STZ, aortic responses to ACh were significantly decreased and shifted to the right in females (Figure 1), and both sensitivity and $\mathrm{E}_{\max }$ to 
ACh were significantly decreased in aorta from female diabetic rats compared with nondiabetic controls (Table 2). Aortic rings from 8-week diabetic males also showed a slight, but insignificant rightward shift of the ACh response. Eight-week STZ-induced diabetes did not affect the sensitivity to ACh in male aortae. However, the $\mathrm{E}_{\max }$ to ACh was significantly reduced compared to that observed in non-diabetic control males (Table 2). The ACh $\mathrm{E}_{\max }$ was $79 \pm 1.8 \%$ in control males and $67 \pm 3.6 \%$ in diabetic males $(n=6$ per group).

An interaction between sex and diabetes was observed in the relaxation responses to $\mathrm{ACh}$ $(P<0.05$, two way ANOVA). When comparing the effect of 8-week STZ-induced diabetes in rightward shifting ACh relaxation in females with that of males, the effect was significantly greater in female compared to males rats as assessed by the bigger shift of ACh response to the right $(P<0.05$, Mann-Whitney test $)$

\section{3) Effects of sex and diabetes on SNP-induced relaxation in rat aortae}

Sensitivity of smooth muscle to NO was assessed by measuring the endotheliumindependent relaxation to SNP $\left(10^{-9}\right.$ to $\left.10^{-5} \mathrm{M}\right)$ in PE-pre-contracted aorta. Although, there was a slight rightward shift of SNP responses in aortic rings from control males relative to control females, there were no significant differences in SNP-induced relaxations between either sexes or diabetic rats and their respective age-matched controls (Figure 2).

\section{4) Effects of sex and diabetes on contractile responses to PE in rat aorta}

To assess whether sex and 8-week diabetes affect the sensitivity and maximal responses to $\alpha$-adrenoceptor_agonist, contractile responses to $\mathrm{PE}\left(10^{-8}\right.$ to $\left.10^{-5} \mathrm{M}\right)$ were 
measured. A sex difference and an interaction between sex and diabetes were shown in the contractile responses to PE. There was a significant leftward shift of PE contractile responses in aortic rings from control males relative to control females (Figure 3). Both maximal tension developed in response to $\mathrm{PE}$ and the sensitivity to $\mathrm{PE}$ in aortic rings from control male rats were significantly higher than those in control females (Table 3 , no drugs (ND)). The PE maximal tension was $1.78 \pm 0.10$ vs. $0.81 \pm 0.14 \mathrm{~g}$, and the $\mathrm{pD}_{2}$ to PE was $7.34 \pm 0.08$ vs. $6.98 \pm 0.05$, in males and females, respectively. Eight-week STZdiabetes shifted PE contractile responses to the left only in the aortic rings of females (Figure 3); both $\mathrm{E}_{\max }$ and the sensitivity to PE were higher in arteries of diabetic females than those in non-diabetic female (Table 3, no drugs (ND)). In males, 8-week STZdiabetes did not affect contractile responses to PE (Table 3, no drugs (ND)).

The administration of indo to block COX metabolites slightly, but significantly, reduced $\mathrm{E}_{\max }$ to $\mathrm{PE}$ only in aorta taken from non-diabetic control females, with in no apparent effect on maximal contractile response in any other experimental groups (Figure 4 and Table 3). After addition of indo, the $\mathrm{PE} \mathrm{E}_{\max }$ was reduced to $68.93 \pm 6.58 \%$ in control females ( $n=6$ per group). Inhibition of COX slightly but significantly reduced the sensitivity to PE in both diabetic and non-diabetic males.

The basal level release of NO was assessed indirectly by measuring the contraction to $\mathrm{PE}\left(10^{-8}\right.$ to $\left.10^{-5} \mathrm{M}\right)$ in aortic rings before and after pre-treatment with LNAME $(200 \mu \mathrm{M}, 20 \mathrm{~min})$ in the presence of indo $(10 \mu \mathrm{M})$. The difference in the contractile level to PE after addition of LNAME would indicate the extent of endothelium NO release (Csanyi et al. 2007; Hayashi et al. 1992). Pretreatment of aortic rings with L-NAME in the presence of indo, significantly increased contractile responses 
to $\mathrm{PE}$ in all four groups (Figure 4). However, $\triangle \mathrm{AUC}$, defined as the difference in area under the curve between PE responses before and after L-NAME, exhibited a greater potentiation of the PE response after NOS inhibition in aortae from control females compared with male controls and diabetic rats, regardless of sex. Interestingly, 8-week STZ diabetes decreased the $\triangle \mathrm{AUC}$ in females to the same level as those seen in males (Table 3).

\section{5) Effects of sex and diabetes on rat aortic eNOS and Nox1 mRNA expression}

To begin investigating the underlying mechanism by which endothelium-derived NO production in response to PE might have been reduced in 8-week STZ diabetes in females, levels of aortic eNOS mRNA expression were determined using real-time PCR. Aortic level of eNOS mRNA expression (Figure 5A) was significantly higher in control females than in control males. Eight weeks STZ-induced diabetes brought the level of eNOS mRNA expression in aortae of females down to the same level as measured in males.

Finally, to assess a potential mechanism for the impairment of ACh response in 8week diabetic rats, the aortic mRNA expression for the Nox subunit (Nox1, a major source of superoxide in aorta) was measured. Real time PCR analysis revealed that the level of mRNA expression for Nox 1 was significantly higher in aortae taken from 8-week STZ-diabetic female rats compared to that observed in non-diabetic control females (Figure 5B). There was also significant difference in aortic Nox 1 mRNA expression level between diabetic female and diabetic male rats. Although Nox1 mRNA tended to 
be greater in 8-week diabetic males than in non-diabetic control males, the difference was not statistically significant.

\section{DISCUSSION}

The current study assessed the influence of both sex and the intermediate stage STZ-induced diabetes on rat aortic endothelial function. We observed that 8 weeks following the induction of diabetes, EDV was impaired to a greater degree in aortic rings from female rats than in males. We also found that the responsiveness to PE was significantly enhanced only in female diabetic rat aortae. Basal NO levels, as indicated by the extent of PE potentiation after L-NAME, was higher in normoglycemic control female rat aortae than that in males. eNOS mRNA expression was also significantly higher in aortae of control females than that in males. However, 8 weeks after the induction of STZ diabetes, both basal NO and eNOS mRNA were reduced in female rat aorta to the same levels seen in males. Accordingly, we observed higher mRNA expression of Nox 1 in aortae of 8-week diabetic females than age-matched control females and diabetic males.

Nitric oxide is considered the major EDRF regulating endothelial and vascular function in large conduit arteries, such as the aorta (Forte et al. 1998; Loria et al. 2014; Vaziri et al. 1998). It is also known that there are sex differences in NO bioavailability and that females have higher levels of NO than males (Forte et al. 1998; GlushkovskayaSemyachkina et al. 2006; Sullivan et al. 2010). When compared NO-dependent responses in intact aortic rings, both enhanced (Aloysius et al. 2012; Gisclard et al. 1988; Rahimian et al. 1997) or no change (Han et al. 2014; Hayashi et al. 1992; Miller and 
Vanhoutte 1991) of EDV to ACh have been reported in female arteries of rats and rabbits compared to male animals. ACh-induced relaxation may be altered by estrous cycle (Liu et al. 2001). Although the phase of the estrous cycle in female rats was not determined in the current study, at the age studied, the EDV to ACh was greater in aortic rings of normoglycemic female than that observed in normoglycemic males suggesting a sex difference in the stimulated release of NO in the rat aorta. This is generally in agreement with numerous studies (Miller 2010; Thompson and Khalil 2003) that demonstrate greater NO in females than males contributes to sex differences in rat aortic vasodilation.

Decreased NO-mediated control of vascular function plays an important role in the development and progression of CVD (Orshal and Khalil 2004; Sader and Celermajer 2002). Decreased EDV has been repeatedly shown in rat aortae from both chemicallyinduced and genetic models of type 1 diabetes (Fukao et al. 1997; Keegan et al. 1995; Miller 2010; Pieper and Siebeneich 1997; Shimizu et al. 1993). In line with previous reports (Fukao et al. 1997; Han et al. 2014; Keegan et al. 1995; Pieper and Siebeneich 1997; Shimizu et al. 1993; Zhang et al. 2012) demonstrate a compromised EDV in different vascular beds in diabetes. Here, we show that 8-week STZ-induced diabetes impairs EDV in rat aorta. We recently reported a sex difference in aortic endothelial function 1 week after the induction of STZ diabetes (Han et al. 2014). In the current study, we observed a greater impairment of EDV to ACh as well as bradykinin (another endothelium-dependent vasodilator, data not shown) in rat aortic rings of female rats than males, 8 weeks following the induction of diabetes. However, when compared with the observations in the 1-week study (Han et al. 2014), the effect of 8-week STZ-induced 
diabetes in blunting EDV in females was much greater than in males as assessed by bigger rightward shift of $\mathrm{ACh}$ response compared to that in the 1-week study (Han et al. 2014). This may suggest that the extent of EDV impairment in female diabetic rat aorta is dependent on the duration of the diabetic state among other factors. This is in agreement with our previous report showing that, in female rats, longer exposure to diabetes leads to a greater impairment of the mesenteric arterial response (Zhang et al. 2012). This may, in part, also support the findings of (Pieper 1999), which showed that the alteration of EDV in diabetes is associated with the duration of the diabetic state (Clarkson et al. 1996; Pieper 1999). However, in contrast with the Piper findings (Pieper 1999), we reported that the impairment of EDV in rat aorta occurred as early as one week after the induction of STZ-diabetes in females (Han et al. 2014).

There are several possible mechanisms that may contribute to the impairment of the EDV to ACh in aortae of 8-week STZ diabetic rats, such as decreased NO bioavailability due to lower production or higher degradation in the endothelial cells, a decreased sensitivity of smooth muscle to $\mathrm{NO}$ and an enhanced vasoconstrictor response to agents such as PE.

The responsiveness of vascular smooth muscle to NO was assessed by measuring aortic relaxation responses to SNP, a NO donor. Despite a slight leftward shift in the relaxation response in control male rats compared to control females, there was no significant difference in the SNP-induced relaxation of the aorta with respect to sex or diabetes. This result suggests that reduced EDV in aorta of diabetic female rats is more likely related to either decreased NO bioavailability or enhanced vasoconstrictor responses. 
We assessed smooth muscle reactivity by measuring $\alpha_{1}$-adrenergic agonist (PE)induced vasoconstriction. Both sensitivity and the maximum contractile response to PE were significantly enhanced in aortic rings from normoglycemic males compared with normoglycemic females. Conflicting data have been reported regarding responses to vasoconstrictor factors in male and female rats. Other investigators reported an increase (Aloysius et al. 2012; Robert et al. 2005), decrease (Li and Stallone 2005), or no change (Loria et al. 2014) in aortic responses to vasoconstrictors in male rats compared with females. The reason for these differences is not clear, but contributing factors may include differences in the type of the vasoconstrictor used, as reported previously (Stallone et al. 1991) as well as the age of the animals used in the various studies.

In experiments examining the effect of age on adrenergic receptor sensitivity, studies revealed that advancing age affects the vasculature of males and females differently (Sullivan and Davison 2001). When comparing the observations in the 1-week study (Han et al. 2014) slightly older normoglycemic female rats became slightly less responsive to $\mathrm{PE}$-induced constriction, whereas constrictor responses in normoglycemic males were slightly increased. This may explain, in part, the sex-related differences observed in ACh-induced relaxation in the PE-pre-contracted aortic rings in the current study.

The decreased responsiveness of female to PE may also be due to a decreased release of contractile factors and/or an enhanced basal NO. Interestingly though, in female rats the incubation of aorta with indo (a COX blocker) slightly, but significantly, attenuated the maximal contractile response to PE, suggesting that contractile factors were slightly enhanced in the female rat aorta (compared to age-matched controls). The indo had no 
effect on the maximal contractile response in aortic rings of control males and diabetic animals, regardless of sex. Along similar lines, Stallone et al., (Stallone et al. 2001) reported that indo reduced the maximal contractile response of female but not male aorta.

In the present study we measured basal NO levels by monitoring the effect of LNAME on PE-induced contraction (Csanyi et al. 2007; Hayashi et al. 1992; Rahimian et al. 2002; Rahimian et al. 1997). Consistent with our previous findings (Han et al. 2014; Rahimian et al. 1997), incubation of aorta rings with L-NAME caused a significantly greater potentiation of the PE response in normoglycemic female rats compared with that observed in other experimental groups, indicating a much higher basal level of NO in female tissues than that in males. Hence, the elevated basal NO may explain the decreased PE responsiveness in female aortae, despite slight increased contractile factors in aorta of this group.

There are also conflicting data regarding the effect of diabetes on the vasoconstrictor responses (Chang and Stevens 1992; Myers and Messina 1996; White and Carrier 1988). Since the ACh-induced aortic relaxation was more impaired in 8-week STZdiabetic female rats, it might be expected that the PE-induced contractile response would be enhanced in the female diabetic group. As predicted, 8-week STZ-induced diabetes resulted in enhanced both the sensitivity and maximal contractile responses to PE in aortic rings of diabetic females (versus its respective control, Figure 3, Table 3). In contrast, no difference in vasoconstrictor responses to PE constriction was found between control and diabetic males, further suggesting a sex-specific response of the aorta. The increased responsiveness of aortic rings from diabetic females to PE may result from elevated release of contractile factors and/or decreased basal NO. Although indo had no 
effect on aortic rings of diabetic females, the level of basal NO, as measured by potentiation of PE responses by L-NAME, was reduced in 8-week diabetic female rats to the same level as those in the male animals. These data are also consistent with our previous report in short-term STZ-induced diabetic female rats (Han et al. 2014).

Decreased NO in aortae from diabetic females may result from a decrease in NO production due to a lower eNOS expression, activity and/or higher NO degradation. We did not directly measure the activity of eNOS. However, our data revealed that 8 weeks after STZ injection, eNOS mRNA levels in female rats was significantly lower than that in the control females. Thus, the impaired responses to $\mathrm{ACh}$ in aortae from 8-week diabetic females may be due, in part, to a decreased NO production.

Finally, impaired EDV could also arise as a result of the inactivation of NO by ROS, such as superoxide. In the cardiovascular system, the Nox family is one of the potent cellular sources of superoxide (Lassegue et al. 2001). Nox1, Nox2, and Nox4 are highly expressed in the vascular wall. However, Nox1 is mainly expressed in large conduit arteries (Lassegue et al. 2001), whereas Nox2 is expressed to a greater degree in resistance arteries (Touyz et al. 2002). We did not measure the levels of superoxide, but 8 weeks after the induction of STZ diabetes the level of Nox1 mRNA expression was upregulated only in female rat aortae, suggesting that an elevation of superoxide may also partially be responsible for the elevated PE contractile responsiveness and further impairment of ACh-dependent relaxation in this group. 


\section{CONCLUSION}

In conclusion, the current study reveals a predisposition of the female rat aorta toward vascular injury in intermediate stage of diabetes ( 8 weeks duration), possibly via an alteration in the synthesis or activity of NO. Furthermore, when compared with the observations in the 1-week study (Han et al. 2014) the magnitude of EDV impairment was significantly greater in 8 -week diabetic female than diabetic males. This study did not examine the impact of sex hormones, particularly estrogen, on diabetes-induced changes in vascular functions as the diabetic female rats were not ovariectomized. Thus, the relevance of the present findings to our understanding of how estrogens affect endothelial function is limited to the experimental model (intact STZ-treated and untreated females and males) that was used. It is also important to consider the role of other contributing factors (sex steroid hormonal milieu and neural factors) on the development of abnormal aortic responses, in addition to that of estrogen. Our current findings do not rule out this possibility. Taken together our data reinforces our hypothesis that sex may influence endothelial function in rat aorta.

\section{Disclosures}

No conflicts of interest, financial or otherwise, are declared by the author(s).

\section{Acknowledgements}

This work was supported, in part, by the National Institutes of Health (NIDCR, DE016587) and the University of the Pacific. We thank Dr. William Bannon from StatsWhisperer for his contribution toward statistical analysis. 


\section{References}

Aloysius, U. I., Achike, F. I., and Mustafa, M. R. 2012. Mechanisms underlining gender differences in Phenylephrine contraction of normoglycaemic and short-term Streptozotocin-induced diabetic WKY rat aorta. Vascul Pharmacol. 57(2-4), 81-90. Bhardwaj, R., and Moore, P. K. 1988. Increased vasodilator response to acetylcholine of renal blood vessels from diabetic rats. J Pharm Pharmacol. 40(10), 739-742.

Cai, H., Griendling, K. K., and Harrison, D. G. 2003. The vascular NAD(P)H oxidases as therapeutic targets in cardiovascular diseases. Trends Pharmacol Sci. 24(9), 471-478. Chang, K. S., and Stevens, W. C. 1992. Endothelium-dependent increase in vascular sensitivity to phenylephrine in long-term streptozotocin diabetic rat aorta. Br J Pharmacol. 107(4), 983-990.

Clarkson, P., Celermajer, D. S., Donald, A. E., Sampson, M., Sorensen, K. E., Adams, M., et al. 1996. Impaired vascular reactivity in insulin-dependent diabetes mellitus is related to disease duration and low density lipoprotein cholesterol levels. J Am Coll Cardiol. 28(3), 573-579.

Csanyi, G., Lepran, I., Flesch, T., Telegdy, G., Szabo, G., and Mezei, Z. 2007. Lack of endothelium-derived hyperpolarizing factor (EDHF) up-regulation in endothelial dysfunction in aorta in diabetic rats. Pharmacol Rep. 59(4), 447-455.

De Vriese, A. S., Verbeuren, T. J., Van de Voorde, J., Lameire, N. H., and Vanhoutte, P. M. 2000. Endothelial dysfunction in diabetes. Br J Pharmacol. 130(5), 963-974.

Faerch, K., Borch-Johnsen, K., Vaag, A., Jorgensen, T., and Witte, D. R. 2010. Sex differences in glucose levels: a consequence of physiology or methodological convenience? The Inter99 study. Diabetologia. 53(5), 858-865. 
Forte, P., Kneale, B. J., Milne, E., Chowienczyk, P. J., Johnston, A., Benjamin, N., et al. 1998. Evidence for a difference in nitric oxide biosynthesis between healthy women and men. Hypertension. 32(4), 730-734.

Fu, G. S., Huang, H., Chen, F., Wang, H. P., Qian, L. B., Ke, X. Y., et al. 2007.

Carvedilol ameliorates endothelial dysfunction in streptozotocin-induced diabetic rats. Eur J Pharmacol. 567(3), 223-230.

Fukao, M., Hattori, Y., Kanno, M., Sakuma, I., and Kitabatake, A. 1997. Alterations in endothelium-dependent hyperpolarization and relaxation in mesenteric arteries from streptozotocin-induced diabetic rats. Br J Pharmacol. 121(7), 1383-1391.

Gisclard, V., Miller, V. M., and Vanhoutte, P. M. 1988. Effect of 17 beta-estradiol on endothelium-dependent responses in the rabbit. J Pharmacol Exp Ther. 244(1), 19-22. Glushkovskaya-Semyachkina, O. V., Anishchenko, T. G., Sindyakova, T. A., Leksina, O. V., and Berdnikova, V. A. 2006. Sex-related differences in nitric oxide content in healthy and hypertensive rats at rest and under stress conditions. Bull Exp Biol Med. 142(1), 9-11. Goel, A., Thor, D., Anderson, L., and Rahimian, R. 2008. Sexual dimorphism in rabbit aortic endothelial function under acute hyperglycemic conditions and gender-specific responses to acute 17beta-estradiol. Am J Physiol Heart Circ Physiol. 294(6), H24112420.

Goel, A., Zhang, Y., Anderson, L., and Rahimian, R. 2007. Gender difference in rat aorta vasodilation after acute exposure to high glucose: involvement of protein kinase $\mathrm{C}$ beta and superoxide but not of Rho kinase. Cardiovasc Res. 76(2), 351-360. 
Gorlach, A., Brandes, R. P., Nguyen, K., Amidi, M., Dehghani, F., and Busse, R. 2000. A gp91phox containing NADPH oxidase selectively expressed in endothelial cells is a major source of oxygen radical generation in the arterial wall. Circ Res. 87(1), 26-32. Griendling, K. K., Sorescu, D., and Ushio-Fukai, M. 2000. NAD(P)H oxidase: role in cardiovascular biology and disease. Circ Res. 86(5), 494-501.

Han, X., Zhang, R., Anderson, L., and Rahimian, R. 2014. Sexual dimorphism in rat aortic endothelial function of streptozotocin-induced diabetes: possible involvement of superoxide and nitric oxide production. Eur J Pharmacol. 723, 442-450.

Hayashi, T., Fukuto, J. M., Ignarro, L. J., and Chaudhuri, G. 1992. Basal release of nitric oxide from aortic rings is greater in female rabbits than in male rabbits: implications for atherosclerosis. Proc Natl Acad Sci U S A. 89(23), 11259-11263.

Ikubo, N., Saito, M., Tsounapi, P., Dimitriadis, F., Ohmasa, F., Inoue, S., et al. 2011. Protective effect of taurine on diabetic rat endothelial dysfunction. Biomed Res. 32(3), 187-193.

Johnstone, M. T., Creager, S. J., Scales, K. M., Cusco, J. A., Lee, B. K., and Creager, M. A. 1993. Impaired endothelium-dependent vasodilation in patients with insulin-dependent diabetes mellitus. Circulation. 88(6), 2510-2516.

Kazuyama, E., Saito, M., Kinoshita, Y., Satoh, I., Dimitriadis, F., and Satoh, K. 2009. Endothelial dysfunction in the early- and late-stage type-2 diabetic Goto-Kakizaki rat aorta. Mol Cell Biochem. 332(1-2), 95-102.

Keegan, A., Walbank, H., Cotter, M. A., and Cameron, N. E. 1995. Chronic vitamin E treatment prevents defective endothelium-dependent relaxation in diabetic rat aorta. Diabetologia. 38(12), 1475-1478. 
Lassegue, B., Sorescu, D., Szocs, K., Yin, Q., Akers, M., Zhang, Y., et al. 2001. Novel gp91(phox) homologues in vascular smooth muscle cells : nox1 mediates angiotensin IIinduced superoxide formation and redox-sensitive signaling pathways. Circ Res. 88(9), 888-894.

Li, M., and Stallone, J. N. 2005. Estrogen potentiates vasopressin-induced contraction of female rat aorta by enhancing cyclooxygenase- 2 and thromboxane function. Am J Physiol Heart Circ Physiol. 289(4), H1542-1550.

Liu, M. Y., Hattori, Y., Fukao, M., Sato, A., Sakuma, I., and Kanno, M. 2001. Alterations in EDHF-mediated hyperpolarization and relaxation in mesenteric arteries of female rats in long-term deficiency of oestrogen and during oestrus cycle. Br J Pharmacol. 132(5), 1035-1046.

Livak, K. J., and Schmittgen, T. D. 2001. Analysis of relative gene expression data using real-time quantitative PCR and the 2(-Delta Delta C(T)) Method. Methods. 25(4), 402408.

Loria, A. S., Brinson, K. N., Fox, B. M., and Sullivan, J. C. 2014. Sex-specific alterations in NOS regulation of vascular function in aorta and mesenteric arteries from spontaneously hypertensive rats compared to Wistar Kyoto rats. Physiol Rep. 2(8). Malhotra, A., Penpargkul, S., Fein, F. S., Sonnenblick, E. H., and Scheuer, J. 1981. The effect of streptozotocin-induced diabetes in rats on cardiac contractile proteins. Circ Res. 49(6), 1243-1250.

Miller, V. M. 2010. Sex-based differences in vascular function. Womens Health (Lond Engl). 6(5), 737-752. 
Miller, V. M., and Vanhoutte, P. M. 1991. Progesterone and modulation of endotheliumdependent responses in canine coronary arteries. Am J Physiol. 261(4 Pt 2), R1022-1027. Myers, T. O., and Messina, E. J. 1996. Depressed arteriolar responsiveness to norepinephrine in streptozotocin-induced diabetes in the rat. Prostaglandins. 52(5), 415 430.

Olukman, M., Sezer, E. D., Ulker, S., Sozmen, E. Y., and Cinar, G. M. 2010. Fenofibrate treatment enhances antioxidant status and attenuates endothelial dysfunction in streptozotocin-induced diabetic rats. Exp Diabetes Res. 2010, 828531.

Orshal, J. M., and Khalil, R. A. 2004. Gender, sex hormones, and vascular tone. Am J Physiol Regul Integr Comp Physiol. 286(2), R233-249.

Pieper, G. M. 1999. Enhanced, unaltered and impaired nitric oxide-mediated endothelium-dependent relaxation in experimental diabetes mellitus: importance of disease duration. Diabetologia. 42(2), 204-213.

Pieper, G. M., and Siebeneich, W. 1997. Diabetes-induced endothelial dysfunction is prevented by long-term treatment with the modified iron chelator, hydroxyethyl starch conjugated-deferoxamine. J Cardiovasc Pharmacol. 30(6), 734-738.

Rahimian, R., Dube, G. P., Toma, W., Dos Santos, N., McManus, B. M., and van Breemen, C. 2002. Raloxifene enhances nitric oxide release in rat aorta via increasing endothelial nitric oxide mRNA expression. Eur J Pharmacol. 434(3), 141-149.

Rahimian, R., Laher, I., Dube, G., and van Breemen, C. 1997. Estrogen and selective estrogen receptor modulator LY117018 enhance release of nitric oxide in rat aorta. J Pharmacol Exp Ther. 283(1), 116-122. 
Robert, R., Chagneau-Derrode, C., Carretier, M., Mauco, G., and Silvain, C. 2005. Gender differences in vascular reactivity of aortas from rats with and without portal hypertension. J Gastroenterol Hepatol. 20(6), 890-894.

Sader, M. A., and Celermajer, D. S. 2002. Endothelial function, vascular reactivity and gender differences in the cardiovascular system. Cardiovasc Res. 53(3), 597-604.

Shen, B., Ye, C. L., Ye, K. H., and Liu, J. J. 2003. Mechanism underlying enhanced endothelium-dependent vasodilatation in thoracic aorta of early stage streptozotocininduced diabetic mice. Acta Pharmacol Sin. 24(5), 422-428.

Shimizu, K., Muramatsu, M., Kakegawa, Y., Asano, H., Toki, Y., Miyazaki, Y., et al. 1993. Role of prostaglandin $\mathrm{H} 2$ as an endothelium-derived contracting factor in diabetic state. Diabetes. 42(9), 1246-1252.

Shimokawa, H., Yasutake, H., Fujii, K., Owada, M. K., Nakaike, R., Fukumoto, Y., et al. 1996. The importance of the hyperpolarizing mechanism increases as the vessel size decreases in endothelium-dependent relaxations in rat mesenteric circulation. $\mathbf{J}$ Cardiovasc Pharmacol. 28(5), 703-711.

Stallone, J. N., Crofton, J. T., and Share, L. 1991. Sexual dimorphism in vasopressininduced contraction of rat aorta. Am J Physiol. 260(2 Pt 2), H453-458.

Stallone, J. N., Salisbury, R. L., and Fulton, C. T. 2001. Androgen-receptor defect abolishes sex differences in nitric oxide and reactivity to vasopressin in rat aorta. J Appl Physiol (1985). 91(6), 2602-2610.

Sullivan, J. C., and Davison, C. A. 2001. Gender differences in the effect of age on electrical field stimulation (EFS)-induced adrenergic vasoconstriction in rat mesenteric resistance arteries. J Pharmacol Exp Ther. 296(3), 782-788. 
Sullivan, J. C., Pardieck, J. L., Hyndman, K. A., and Pollock, J. S. 2010. Renal NOS activity, expression, and localization in male and female spontaneously hypertensive rats. Am J Physiol Regul Integr Comp Physiol. 298(1), R61-69.

Thompson, J., and Khalil, R. A. 2003. Gender differences in the regulation of vascular tone. Clin Exp Pharmacol Physiol. 30(1-2), 1-15.

Touyz, R. M., Chen, X., Tabet, F., Yao, G., He, G., Quinn, M. T., et al. 2002. Expression of a functionally active gp91phox-containing neutrophil-type NAD(P)H oxidase in smooth muscle cells from human resistance arteries: regulation by angiotensin II. Circ Res. 90(11), 1205-1213.

Vaziri, N. D., Ni, Z., and Oveisi, F. 1998. Upregulation of renal and vascular nitric oxide synthase in young spontaneously hypertensive rats. Hypertension. 31(6), 1248-1254. White, R. E., and Carrier, G. O. 1988. Enhanced vascular alpha-adrenergic neuroeffector system in diabetes: importance of calcium. Am J Physiol. 255(5 Pt 2), H1036-1042. Zhang, R., Thor, D., Han, X., Anderson, L., and Rahimian, R. 2012. Sex differences in mesenteric endothelial function of streptozotocin-induced diabetic rats: a shift in the relative importance of EDRFs. Am J Physiol Heart Circ Physiol. 303(10), H1183-1198. 
Table 1. Body weight and blood glucose levels of male and female rats at 8 weeks after vehicle or STZ injection.

\begin{tabular}{cccc}
\hline Group & $\boldsymbol{n}$ & Weight $(\mathbf{g})$ & Blood Glucose (mg/dl) \\
\hline Control Female & 9 & $252.8 \pm 3.4$ & $161 \pm 29$ \\
Diabetic Female & 5 & $211.5 \pm 14.8$ & $515 \pm 67^{\mathrm{b}}$ \\
Control Male & 6 & $396.8 \pm 11.9^{\mathrm{a}}$ & $124 \pm 12$ \\
Diabetic Male & 6 & $310.5 \pm 28.3^{\mathrm{ab}}$ & $497 \pm 66^{\mathrm{b}}$ \\
\hline
\end{tabular}

Data are expressed as mean \pm SEM.

$P<0.05$ female vs male, diabetes vs control (Weight); $P<0.05$ diabetes vs control (Blood Glucose)

${ }^{\mathrm{a}} P<0.05$ (vs. female in the respective treatment); ${ }^{\mathrm{b}} P<0.05$ (vs. non-diabetic, same sex), analyzed using two-way ANOVA followed by LSD post hoc test. 
Table 2. $\mathrm{pD}_{2}$ and $\mathrm{E}_{\max }$ to acetylcholine $(\mathrm{ACh})$ of male and female rats at 8 weeks after vehicle or STZ injection.

\begin{tabular}{cccc}
\hline Group & $\boldsymbol{N}$ & $\mathbf{p D}_{\mathbf{2}}$ & $\mathbf{E}_{\max }(\mathbf{\%})$ \\
\hline Control Female & 9 & $7.47 \pm 0.09$ & $89 \pm 1.3$ \\
Diabetic Female & 5 & $7.16 \pm 0.10^{\mathrm{b}}$ & $63 \pm 6.7^{\mathrm{b}}$ \\
Control Male & 6 & $7.13 \pm 0.06^{\mathrm{a}}$ & $79 \pm 1.8^{\mathrm{a}}$ \\
Diabetic Male & 6 & $7.07 \pm 0.04$ & $67 \pm 3.6^{\mathrm{b}}$ \\
\hline
\end{tabular}

Data are expressed as mean \pm SEM. $\mathrm{pD}_{2}:-\log \mathrm{EC}_{50}$.

$P<0.05$ female vs male, diabetes vs control $\left(\mathrm{pD}_{2}\right) ; \quad P<0.05$ diabetes vs control $\left(\mathrm{E}_{\max }\right)$

${ }^{\mathrm{a}} P<0.05$ (vs. female in the respective treatment); ${ }^{\mathrm{b}} P<0.05$ (vs. non diabetic, same sex), analyzed using two-way ANOVA followed by LSD post hoc test. 
Table 3. $\mathrm{E}_{\max }$, Tension $_{\max }, \mathrm{pD}_{2}$ and $\triangle \mathrm{AUC}$ to phenylephrine (PE) with no drug (ND), indomethacin (Indo), or Indo+L-NAME in male and female rat aortae at 8 weeks after vehicle or STZ injection.
n $\quad \mathbf{E}_{\max }(\%)$
Tension $_{\max }$
$\mathbf{p D}_{2}$
$\triangle \mathbf{A U C}$

(g)

Control Female 6

$\begin{array}{lcccc}\text { ND } & 100 & 0.81 \pm 0.14 & 6.98 \pm 0.05 & \\ \text { Indo } & 68.93 \pm 6.58^{\mathrm{c}} & 0.58 \pm 0.14^{\mathrm{c}} & 7.08 \pm 0.08 & - \\ & & & \\ \text { Indo+LNAME } & 233.86 \pm 16.04^{\mathrm{cd}} & 1.83 \pm 0.26^{\mathrm{cd}} & 7.50 \pm 0.07^{\mathrm{cd}} & 394.25 \pm 48.78\end{array}$

Diabetic female

5

$\begin{array}{lcccc}\text { ND } & 100 & 1.35 \pm 0.08^{\mathrm{b}} & 7.28 \pm 0.06^{\mathrm{b}} & \\ & 85.61 \pm 9.84 & 1.18 \pm 0.19^{\mathrm{b}} & 7.16 \pm 0.08 & - \\ \text { Indo } & 166.86 \pm 12.16^{\mathrm{bcd}} & 2.21 \pm 0.10^{\mathrm{cd}} & 7.94 \pm 0.16^{\mathrm{cd}} & 231.96 \pm 43.32^{\mathrm{f}}\end{array}$

Control Male 6

\begin{tabular}{|c|c|c|c|c|}
\hline ND & 100 & $1.78 \pm 0.10^{\mathrm{a}}$ & $7.34 \pm 0.08^{\mathrm{a}}$ & \\
\hline Indo & $88.01 \pm 6.40$ & $1.58 \pm 0.19^{\mathrm{a}}$ & $7.13 \pm 0.08^{c}$ & - \\
\hline Indo+LNAME & $134.91 \pm 8.29^{\mathrm{acd}}$ & $2.39 \pm 0.16^{\mathrm{acd}}$ & $7.83 \pm 0.19^{\mathrm{cd}}$ & $151.35 \pm 33.10^{\mathrm{e}}$ \\
\hline
\end{tabular}

Diabetic Male 6

$\begin{array}{lcccc}\text { ND } & 100 & 1.69 \pm 0.18^{\mathrm{a}} & 7.26 \pm 0.11 & \\ \text { Indo } & 94.65 \pm 3.10 & 1.76 \pm 0.27 & 7.11 \pm 0.11^{\mathrm{c}} & - \\ & & & & \\ \text { Indo+LNAME } & 153.81 \pm 23.78^{\mathrm{cd}} & 2.51 \pm 0.24^{\mathrm{cd}} & 8.02 \pm 0.16^{\mathrm{cd}} & 196.47 \pm 28.31\end{array}$

Data are expressed as mean \pm SEM. $\triangle \mathrm{AUC}$ : differences of area under the concentrationresponse curve with or without L-NAME in the presence of indo. 
${ }^{\mathrm{a}} P<0.05$ (vs. female in the respective treatment); ${ }^{\mathrm{b}} P<0.05$ (vs. non diabetic, same sex); ${ }^{\mathrm{c}}$ $P<0.05$ (vs. ND); ${ }^{\mathrm{d}} P<0.05$ (vs. indo), analyzed using three-way ANOVA followed by LSD post hoc test.

$P<0.05$ female vs male, interaction between sex and diabetes $(\triangle \mathrm{AUC})$

${ }^{\mathrm{e}} P<0.05$ (vs. female in the respective treatment); ${ }^{\mathrm{f}} P<0.05$ (vs. non diabetic, same sex), analyzed using two-way ANOVA followed by LSD post hoc test. 
Figure 1. Relaxation response to cumulative concentrations of acetylcholine (ACh) in intact aortic rings pre-contracted with phenylephrine (PE, $2 \mu \mathrm{M})$ from female and male rats eight weeks after vehicle or STZ treatment. Relaxation to ACh is expressed as a percentage of PE $(2 \mu \mathrm{M})$ maximum contraction. Data are expressed as mean $\pm \mathrm{SEM}$. $P<0.05$ female vs male; diabetes vs control; interaction between sex and diabetes $* P<0.05$, two-way ANOVA followed by LSD post hoc test. \# $P<0.05$ between male and female rats using Mann-Whitney nonparametric test.

Figure 2. Relaxation response to cumulative concentrations of sodium nitroprusside (SNP) in intact aortic rings pre-contracted with phenylephrine (PE, $2 \mu \mathrm{M})$ from male and female rats at 8 week after vehicle or STZ treatment. Relaxation to SNP is expressed as a percentage of PE $(2 \mu \mathrm{M})$ maximum contraction. Data are expressed as mean $\pm \mathrm{SEM}$.

Figure 3. Contractile response to cumulative concentrations of phenylephrine (PE) in intact aortic rings from female and male rats at 8 week after vehicle or STZ treatment. Data are expressed as mean \pm SEM. $P<0.05$ female vs male; interaction between sex and diabetes

$* P<0.05$, two-way ANOVA followed by LSD post hoc test.

Figure 4. Contractile response to cumulative concentrations of phenylephrine (PE) in intact aortic rings from control female (A), diabetic female (B), control male (C) and diabetic male (D) rats at 8 week after vehicle or STZ treatment. Contraction to PE was measured in absence of any drugs (ND) or in presence of indomethacin (indo: $10 \mu \mathrm{M}$ ) 
followed by addition of $\mathrm{N}^{\omega}$-Nitro-L-arginine methyl ester (indo + L-NAME, $200 \mu \mathrm{M}$ ).

Data are expressed as mean \pm SEM. Repeated measures ANOVA followed by LSD post hoc test: * ND vs. indo $(p<0.05)$ or ND vs. indo + L-NAME $(p<0.05)$; \# indo vs. indo + L-NAME $(p<0.05)$.

Figure 5. Real-time PCR analysis of eNOS (A) and Nox1 (B) mRNA expression in rat thoracic aortae of male and female rats at 8 week after vehicle or STZ treatment. Data are expressed as mean \pm SEM. The eNOS and Nox1 mRNA expression levels of control female rats were normalized as one. $P<0.05$ diabetes vs control (eNOS and Nox1); female vs male (eNOS)

Capped lines indicate statistical differences $(P<0.05, n=5-6$ /group) using two-way ANOVA followed by LSD post hoc test. 


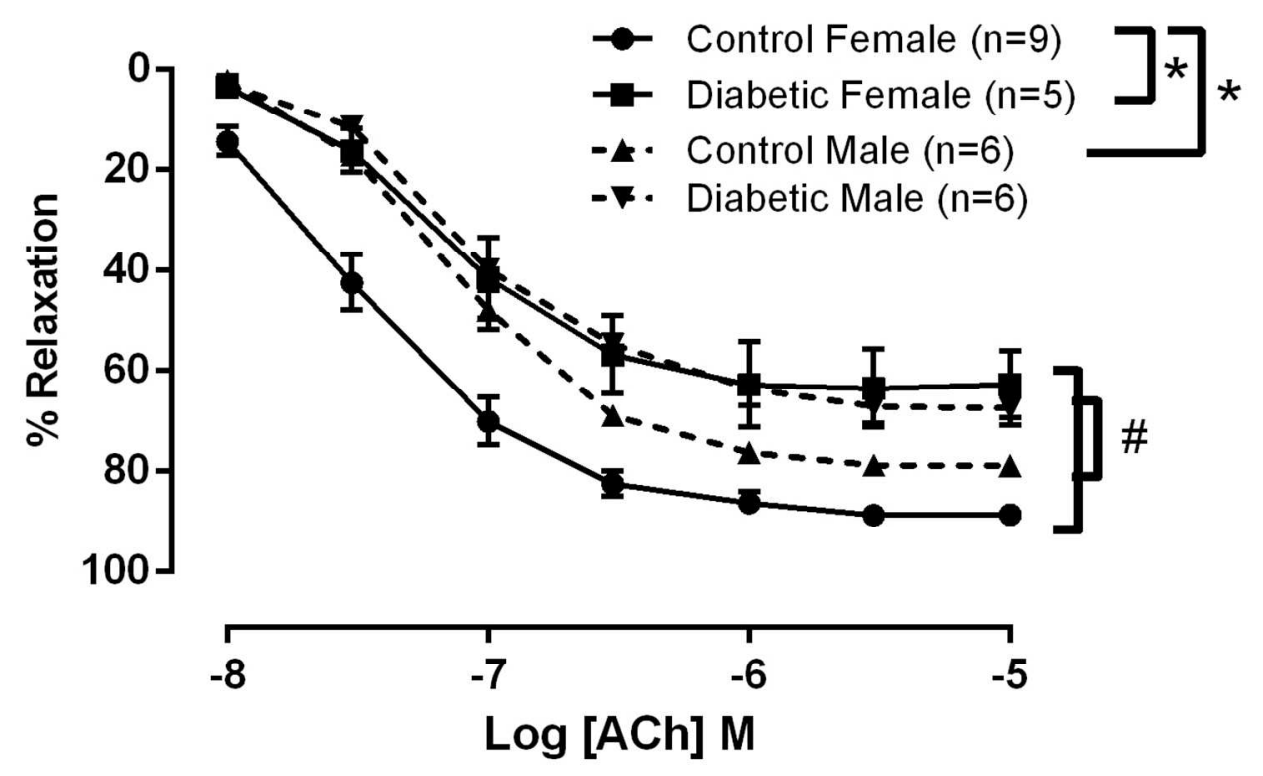

$118 \times 74 \mathrm{~mm}(300 \times 300 \mathrm{DPI})$ 


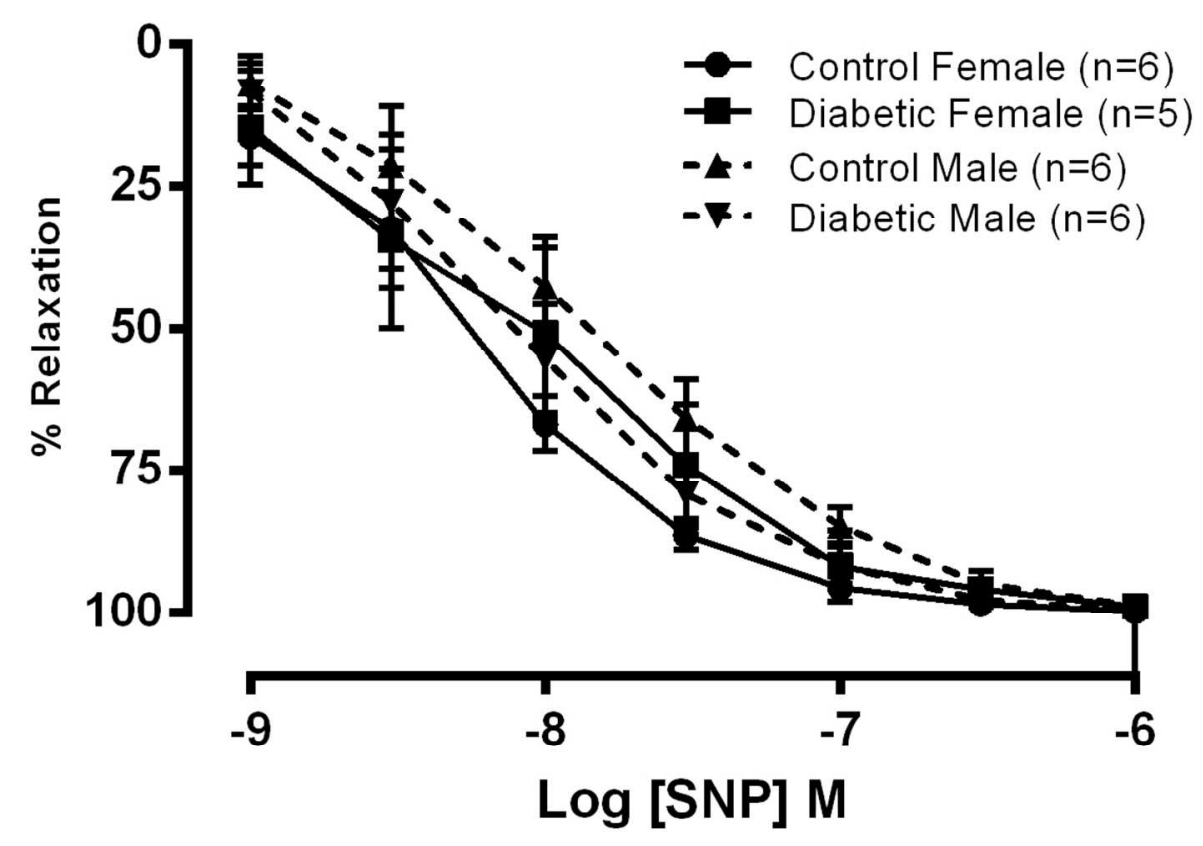

$104 \times 73 \mathrm{~mm}(300 \times 300$ DPI $)$ 


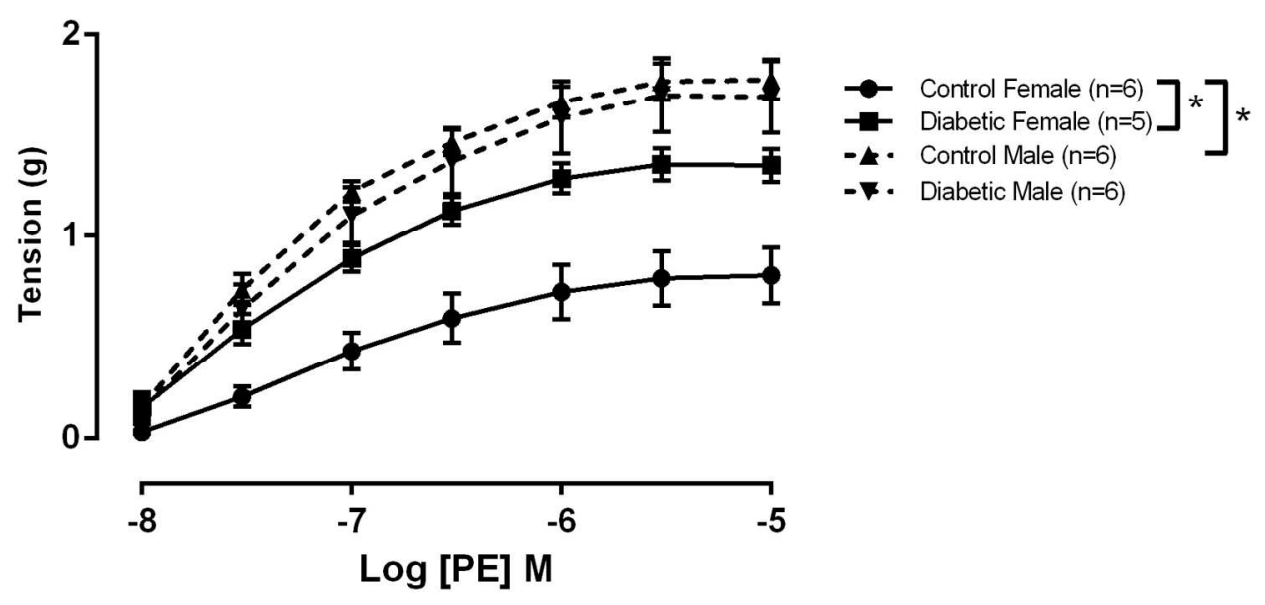

$146 \times 73 \mathrm{~mm}(300 \times 300$ DPI $)$ 
A) Control Female $(n=6)$

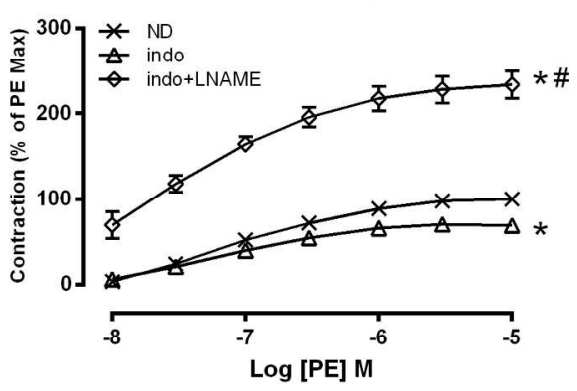

C) Control Male $(n=6)$

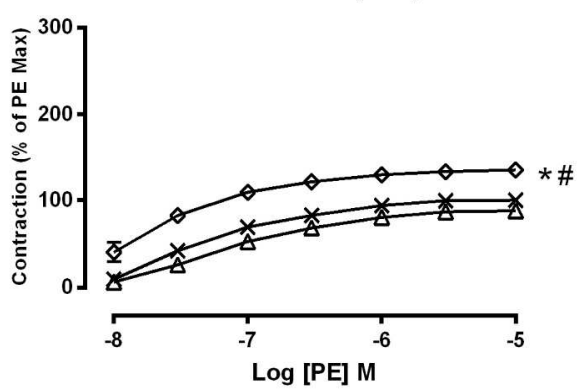

B) Diabetic Female $(n=5)$

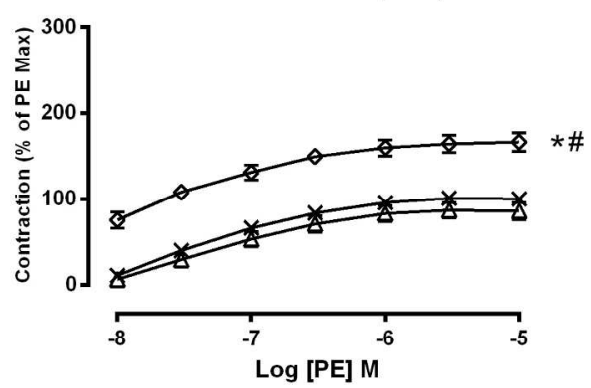

D) Diabetic Male $(\mathrm{n}=6)$

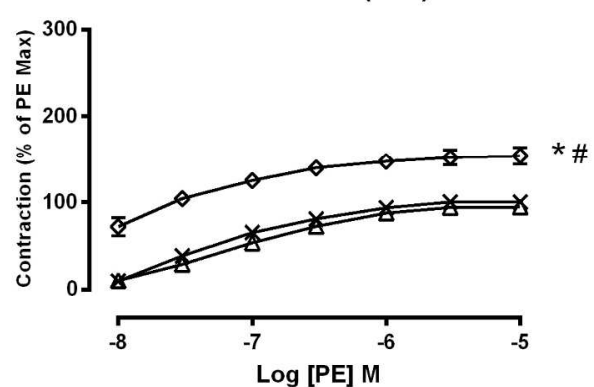

$184 \times 137 \mathrm{~mm}(300 \times 300$ DPI $)$ 

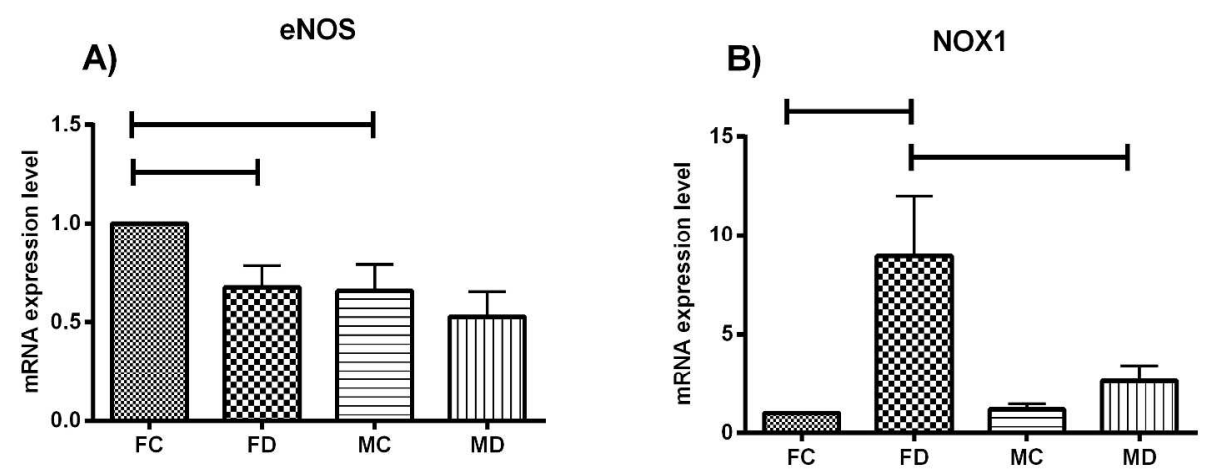

$260 \times 106 \mathrm{~mm}(300 \times 300$ DPI $)$ 